\title{
Understanding fluid transport through the multiscale pore network of a natural shale
}

\author{
Catherine A. Davy ${ }^{1, \star}$, Thang Nguyen Kim ${ }^{2}$, Yang Song ${ }^{3}$, David Troadec $^{4}$, Anne-Marie Blanchenet ${ }^{5}$, and \\ Pierre M. Adler² \\ ${ }^{1}$ Laboratoire de Mécanique de Lille (LML), FRE CNRS 3723 and Ecole Centrale de Lille, CS 20048, \\ F-59651 Villeneuve d'Ascq Cedex, France \\ 2 Metis UMR CNRS 7619, UPMC, Paris, France \\ ${ }^{3}$ Changzhou Institute of Technology, Chang Zhou Gong Xue Yuan, Changzhou Shi, Jiangsu Sheng, China, 213000 \\ 4 Institut d'Electronique, de MicroElectronique et de Nanotechnologie (IEMN), UMR CNRS 8520, \\ BP60069, 59652 Villeneuve d'Ascq, France \\ 5 Unité Matériaux et Transformations (UMET), UMR CNRS 8207, Université Lille 1, Bâtiment C6, \\ 59655 Villeneuve d'Ascq, France
}

\begin{abstract}
The pore structure of a natural shale is obtained by three imaging means. Micro-tomography results are extended to provide the spatial arrangement of the minerals and pores present at a voxel size of $700 \mathrm{~nm}$ (the macroscopic scale). FIB/SEM provides a 3D representation of the porous clay matrix on the so-called mesoscopic scale (10-20 nm); a connected pore network, devoid of cracks, is obtained for two samples out of five, while the pore network is connected through cracks for two other samples out of five. Transmission Electron Microscopy (TEM) is used to visualize the pore space with a typical pixel size of less than $1 \mathrm{~nm}$ and a porosity ranging from 0.12 to 0.25 . On this scale, in the absence of $3 \mathrm{D}$ images, the pore structure is reconstructed by using a classical technique, which is based on truncated Gaussian fields. Permeability calculations are performed with the Lattice Boltzmann Method on the nanoscale, on the mesoscale, and on the combination of the two. Upscaling is finally done (by a finite volume approach) on the bigger macroscopic scale. Calculations show that, in the absence of cracks, the contribution of the nanoscale pore structure on the overall permeability is similar to that of the mesoscale. Complementarily, the macroscopic permeability is measured on a centimetric sample with a neutral fluid (ethanol). The upscaled permeability on the macroscopic scale is in good agreement with the experimental results.
\end{abstract}

\section{Introduction}

\subsection{Scientific context}

Claystones and shales are fine grained sedimentary rocks. Their pores are mainly located within their clay matrix or at its interface with non porous minerals [3-6, 10, 14]. The clay pores, of $1-100 \mathrm{~nm}$ size ${ }^{1}$, most likely dominate the flow and transport properties $[5,11,12]$. Moreover, during sedimentation and compaction, clay minerals are deposited along preferred orientations, i.e. natural shales are generally anisotropic [7]. It is expected that the pore space geometry is also anisotropic, as are the transport properties of the shale [15].

The pore structure of claystones is still partly understood, both in terms of geometry and connectivity, though significant progress has been made over the last decade $[3-5,10-12,14]$. The inherent heterogeneity of these natural materials (which comprise swelling clay, soft and hard

\footnotetext{
^e-mail: catherine.davy@ centralelille.fr

${ }^{1}$ In all this contribution, unless otherwise stated, pore size refers to pore diameter and not radius.
}

minerals), and the broad range of pore sizes, makes them complex to characterize. At least for the natural shale studied herein, at present, no technique alone provides a full overview of its porosity for bulk matter at the centimetric scale and above, due to its very wide pore range. A combination of techniques is usually preferred. They are either direct, i.e. imaging techniques, or indirect, i.e. they require a model to derive pore characteristics. Indirect techniques often rely either on gas adsorption (for the smallest nanoscopic pores) or on capillary intrusion (for pluri-nanometric to millimetric pores). The sample preparation technique may also limit the applicability to bulk matter.

The 3D characterization of the pore structure by direct imaging provides all necessary geometrical parameters, but this largely depends on the pore and sample size considered. Currently, X-Ray micro-tomography (microCT) provides data of the $3 \mathrm{D}$ pore space down to about 700 $\mathrm{nm}$. This is insufficient for clay rocks, which display no connected porosity on this scale, although the analyzed sample sizes are representative of a "macroscopic" struc- 
ture (up to about 180 microns) [10]. Higher resolutions are available with Focused Ion Beam/Scanning Electron Microscopy (FIB/SEM) down to 10-20 nm voxel size, and Transmission Electron Microscopy (TEM) or Scanning Transmission Electron Microscopy (STEM) techniques, down to below $1 \mathrm{~nm}$ pixel/voxel size. However, analyzing one single sample with these techniques does not provide macroscopic representativeness due to the limited sample size. In practice, the representativeness issue is solved by (1): characterizing the sole clay matrix with FIB/SEM and (S)TEM on several samples to achieve representativeness in a statistical sense [2, 5], and by (2): coupling these with micro-CT using scale change methods [8]. Where no single characterization method provides adequate representativeness for such wide pore scales as in clay rocks, the combination of micro-CT, FIB/SEM and TEM is able to provide a full representative $3 \mathrm{D}$ pore space [2].

In this contribution, we benefit from synthetic images derived by training image techniques from micro-CT data for the natural shale from [10]. We also combine both FIB/SEM and TEM imaging of the mesoscopic clay matrix to describe the smallest $1-100 \mathrm{~nm}$ pores. Four percolating FIB/SEM samples $\left(27.8-146.7 \mu \mathrm{m}^{3}\right)$, and up to eight TEM images $\left(18.5 \times 10^{3}-41.0 \times 10^{3} \mathrm{~nm}^{2}\right)$ are used, which are fully analyzed in previous research $[11,12]$.

\subsection{Aims and scopes}

The images are used as a starting point for numerical simulations on all three scales. At the nanoscopic scale given by $2 \mathrm{D}$ TEM, in the absence of $3 \mathrm{D}$ images, the pore structure is reconstructed by using a technique, which is based on truncated Gaussian fields. Permeability calculations are performed with the Lattice Boltzmann Method (LBM) on the mesoscale given by 3D FIB/SEM pore structures, and also on the nanoscale, and on the combination of the two. Upscaling is finally done (by a finite volume approach) at the macroscopic scale by using synthetic images obtained by direct sampling from actual micro-tomography images of the same shale [13]. Complementarily, the macroscopic permeability is measured on a centimetric sample with a neutral fluid (ethanol). The upscaled permeability at the macroscopic scale is compared with the experimental results.

\section{Methods}

\subsection{Experimental}

For FIB/SEM or TEM imaging, two shale samples EST26095 and EST25679 are oven-dried at $80^{\circ} \mathrm{C}$ for at least 15 days and impregnated with non radioactive Methyl MethAcrylate resin (MMA) under partial vaccuum. Following impregnation, MMA is polymerized at a temperature of $55^{\circ} \mathrm{C}$ for $16 \mathrm{~h}$. Prior to polymerization, MMA has a very low viscosity (of $0.6 \mathrm{cP}$ at $20^{\circ} \mathrm{C}$ ) smaller than that of water $\left(1.0020 \mathrm{cP}\right.$ at $\left.20^{\circ} \mathrm{C}\right)$. Therefore, it has excellent pore filling ability. Moreover, for further polishing, polymerized MMA (PMMA) limits the hardness difference between the paste and the aggregates, and proper flatness is
Table 1. Overview of the 3D FIB/SEM samples (1)

\begin{tabular}{lll}
\hline Sample & EST27405-1 & EST27405-2 \\
\hline $\begin{array}{l}\text { Sample orientation } \\
\text { wrt. bedding planes }\end{array}$ & perpendicular & perpendicular \\
Voxel size $\left(\mathrm{nm}^{3}\right)$ & $8.49 \times 10.78 \times 10$ & $5.94 \times 7.54 \times 10$ \\
Volume $\left(\mu \mathrm{m}^{3}\right)$ & $6.55 \times 7.97 \times 1.03$ & $3.99 \times 5.95 \times 1.17$ \\
$\begin{array}{l}\text { Porosity }(\%) \\
\begin{array}{l}\text { Surface connected } \\
\text { orientation }\end{array}\end{array}$ & $\mathbf{z}$ & 4.89 \\
\hline
\end{tabular}

Table 2. Overview of the 3D FIB/SEM samples (2)

\begin{tabular}{llll}
\hline Sample & EST26095-1 & EST26095-2 & EST26095-3 \\
\hline $\begin{array}{l}\text { Orientation } \\
\text { wrt. bedding } \\
\text { planes }\end{array}$ & parallel & parallel & parallel \\
Voxel size & $8.48 \times 10.77$ & $8.48 \times 10.77$ & $8.49 \times 10.78$ \\
$\left(\mathrm{~nm}^{3}\right)$ & $\times 20$ & $\times 20$ & $\times 20$ \\
Volume & $6.55 \times 5.21$ & $6.30 \times 6.99$ & $4.50 \times 6.22$ \\
$\left(\mu \mathrm{m}^{3}\right)$ & $\times 4.30$ & $\times 2.02$ & $\times 3.98$ \\
$\begin{array}{l}\text { Porosity }(\%) \\
\text { Percolating } \\
\text { direction }\end{array}$ & $\mathbf{y}$ & 5.9 & 5.21 \\
\hline
\end{tabular}

Table 3. Characteristics of the TEM images used for the numerical simulations of transport.

\begin{tabular}{lllll}
\hline Sample & $\begin{array}{l}\text { Pixel } \\
\text { size }\left(\mathrm{nm}^{2}\right)\end{array}$ & $\begin{array}{l}\text { Sample } \\
\text { size }\left(\mathrm{nm}^{2}\right)\end{array}$ & $\begin{array}{l}\text { Crop } \\
\text { size }\left(\mathrm{nm}^{2}\right)\end{array}$ \\
\hline $\mathrm{T} 10_{1}$ & $0.36 \times 0.36$ & $658 \times 799$ & $176 \times 193$ & 0.1 \\
$\mathrm{~T}_{2}$ & $0.85 \times 0.85$ & $1540 \times 1886$ & $140 \times 132$ & 0.142 \\
$\mathrm{~T} 09_{1}$ & $1.04 \times 1.04$ & $1903 \times 2329$ & $160 \times 240$ & 0.25 \\
\hline
\end{tabular}

achieved more smoothly than without any impregnation. For FIB/SEM observations, a third core origin EST27405 is used after $60^{\circ} \mathrm{C}$ oven-drying for at least 15 days, but without PMMA impregnation. For TEM imaging, the EST25679 sample is thinned to several tens of microns, prior to being ion milled using a Gatan Duomill ${ }^{T M}$ apparatus, with the use of a liquid nitrogen-cooled specimen holder, until sample edges show electron transparency. This corresponds to thicknesses below $100 \mathrm{~nm}$.

All grayscale images acquired by FIB/SEM and TEM are filtered, thresholded and binarized, to distinguish between the pores and the solids. For FIB/SEM, the direction perpendicular to imaging is $\mathbf{z}$ (it is the direction for slicing the sample at 10 to $20 \mathrm{~nm}$ thickness), whereas each image is in a $(\mathbf{x}, \mathbf{y})$ plane. Full details on the procedures are given in $[11,12]$. The main properties of the samples are gathered in Tables 1 and 2.

\subsection{Numerical}

On the nanoscopic scale, in the absence of 3D images, the pore structure is reconstructed from the 2D TEM images, with the same porosity and the same correlation function, by using a technique based on truncated Gaussian fields 


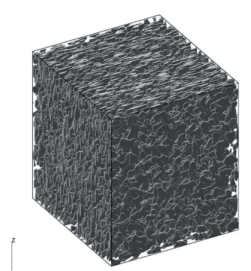

Figure 1. Example of a 3D reconstructed pore structure from the 2D TEM image $\mathrm{T}_{2} 8_{2}$ (porosity 0.142 , $l_{x}=6, l_{y}=l_{z}=50$ ), for a volume of $451 \times 419 \times 484$ pixels $^{3}$ and pixel size $a=2.12 \mathrm{~nm}$.

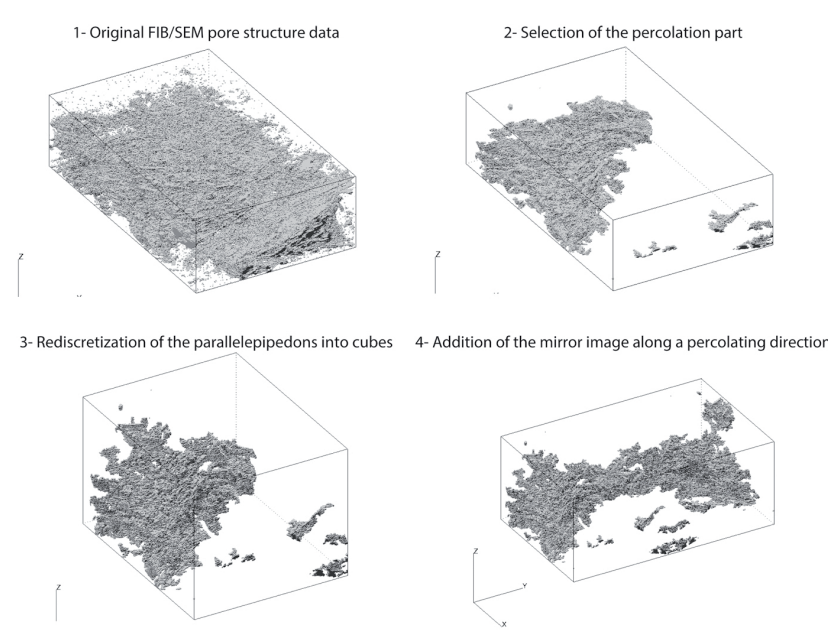

Figure 2. Numerical method providing a cubic and periodic pore volume from 1) the FIB/SEM image of EST26095-1. 2) The percolating part of the pore structure is retained. 3) The parallelepipedic voxels are rediscretized into cubes. 4) The mirror image of the percolating pore structure is added along a percolating direction.

[1]. A correlation function of the following form:

$$
R_{Y}(\mathbf{u})=R_{Y}\left(u_{x}, u_{y}, u_{z}\right)=\exp \left\{-\left(u_{x} / l_{x}\right)^{2}-\left(u_{y} / l_{y}\right)^{2}-\left(u_{z} / l_{z}\right)^{2}\right\}
$$

where $l_{x}<l_{y}=l_{z}$ is used to generate flat-shaped nanopores, i.e. oblates (Fig. 1). It is assumed that directions $\mathbf{y}$ and $\mathbf{z}$ are equivalent.

On both the nanoscopic and mesoscopic scales, the permeability is calculated by solving the Stokes equation on the $3 \mathrm{D}$ pore structures. The same single phase Lattice Boltzmann code is used. According to the standard terminology, it is a D3Q19 code with two relaxation times. The classical bounce-back condition is used at the solid interface. For FIB/SEM data, each sample is completed by its mirror image along a percolating direction, which provides a periodic volume, in order to avoid overall boundary effects (Fig. 2). By construction, the 3D pore volume at the nanoscopic scale is periodic. More details can be found in [9].

\section{Results and discussion}

\subsection{Permeability at the nanoscale}

The dimensionless permeability $K_{n} / a^{2}$ (where $a$ is the cubic voxel size) is determined along each spatial direction $\mathbf{x}, \mathbf{y}$ and $\mathbf{z}$ (these are the $K_{n x x}, K_{n y y}$ and $K_{n z z}$ parameters) as a function of porosity $\varepsilon$ (Table 4 ). In absolute values, the permeability at the nanoscale varies between $0.7 \times 10^{-20} \mathrm{~m}^{2}$ and $1.8 \times 10^{-19} \mathrm{~m}^{2}$. As expected, an
Table 4. Dimensionless permeability on the nanoscale. Cubic voxel size $a: 2.12 \mathrm{~nm}$.

\begin{tabular}{lllll}
\hline Porosity & Percolation & & & \\
$\varepsilon$ & axis & $K_{n x x} / a^{2}$ & $K_{n y y} / a^{2}$ & $K_{n z z} / a^{2}$ \\
\hline 0.102 & none & - & - & - \\
0.130 & $\mathbf{y}$ only & 0 & $0.113 \times 10^{-2}$ & $0.65 \times 10^{-3}$ \\
0.142 & $\mathbf{x}, \mathbf{y}, \mathbf{z}$ & $0.487 \times 10^{-2}$ & $0.147 \times 10^{-2}$ & $=K_{n y y} / a^{2}$ \\
0.200 & $\mathbf{x}, \mathbf{y}, \mathbf{z}$ & $0.686 \times 10^{-2}$ & $0.138 \times 10^{-1}$ & $=K_{\text {nyy }} / a^{2}$ \\
0.252 & $\mathbf{x}, \mathbf{y}, \mathbf{z}$ & $0.802 \times 10^{-2}$ & $0.404 \times 10^{-1}$ & $=K_{n y y} / a^{2}$ \\
\hline
\end{tabular}

Table 5. Dimensionless permeability on the mesoscale.

\begin{tabular}{lllll}
\hline $\begin{array}{l}\text { Sample } \\
\mathrm{n} .\end{array}$ & $\begin{array}{l}\text { Cubic } \\
\text { voxel } \\
\text { size } a(\mathrm{~nm})\end{array}$ & $\begin{array}{l}K_{m y y} / a^{2} \\
\left(\times 10^{-2}\right)\end{array}$ & $\begin{array}{l}K_{m z z} / a^{2} \\
\left(\times 10^{-2}\right)\end{array}$ & $\begin{array}{l}K_{m} \\
\left(10^{-20}\right. \\
\left.\mathrm{m}^{2}\right)\end{array}$ \\
\hline EST26095-1 & 8.48 & 0.112 & 0 & 8.05 \\
EST26095-2 & 8.48 & 0 & 0.0421 & 3.03 \\
EST27405-1 & 8.48 & 0 & 0.379 & 27.3 \\
EST27405-2 & 5.94 & 0 & 3.40 & 120 \\
\hline
\end{tabular}

anisotropy exists between the axes $\mathbf{x}$ and $\mathbf{y}$ (i.e. $\mathbf{z}$ also), as $K_{n z z}$ is consistently greater than $K_{n x x}$.

\subsection{Permeability at the mesoscale}

On the mesoscale, percolation of the FIB/SEM pore volumes occurs only along one direction, either $\mathbf{z}$ (for EST27405-1, EST27405-2 and EST26095-2) or $\mathbf{y}$ (for EST26095-1). The mesoscopic permeability $K_{m}$ varies between $3.3 \times 10^{-20} \mathrm{~m}^{2}$ and $1.20 \times 10^{-18} \mathrm{~m}^{2}$, depending on the nature of the percolating volume (Table 5). The biggest $K_{m}$ values, obtained with EST27405-1 and EST27405-2, correspond to percolating volumes identified as cracks [12].

\subsection{Permeability by combining the nanoscale and the mesoscale pore structures}

First, it is assumed that the permeability $K_{n m}$ of the superposed nanoscale and mesoscale porosities is on the order of $K_{n}$, i.e. $K_{n m} \approx K_{n}$. Due to current limitations of the calculation means, the mesoscopic pore volume is subsampled and superposed to the nanoscopic pore volume.

For instance, for the FIB/SEM volume EST274051 , a sub-sample provides an dimensionless permeability $K_{m z z} / a^{2}$ of 3.77 , whereas the same volume superposed with the nanoscopic pore volume of porosity 0.142 provides a $K_{n m z z} / a^{2}=3.92$. For nanoscale porosity values ranging between 0.102 and $0.252, K_{n m z z} / a^{2}$ ranges between 3.87 and 4.14. In this case of a cracked mesoscopic sample, the difference between $K_{n m}$ and $K_{m}$ is very limited.

A second sub-sample of the same mesoscopic pore volume provides $K_{m z z} / a^{2}=0.133 \times 10^{-1}$ (alone), whereas the nanoscopic pore volume of porosity 0.2 (and correlation lengths of $1.5,12.5$ and $12.5 \mathrm{~nm}$ ) provides $K_{n z z} / a^{2}=0.489 \times 10^{-2}$ (alone). The combination of the two provides $K_{n m z z} / a^{2}=0.236 \times 10^{-1}$, which is significantly greater than $K_{m z z} / a^{2}$ alone. This means that the 
nanoscopic pore structure cannot be neglected to estimate the shale overall permeability.

\subsection{Permeability at the macroscopic scale}

On this scale, a finite volume approach is preferred. It is performed on synthetic 3D images, obtained by the direct sampling method [13] on actual micro-tomography images of the same shale as in [11]. The medium is composed of three phases. Phase 1 corresponds to the macropores (volume percentage 0.5 ), with an infinite permeability $K_{\text {pore }}=\infty$; for practical reasons, $K_{\text {pore }}$ is at given values of $0,1,10,10^{2}, 10^{3}, 10^{4}$ and $10^{5}$; Phase 2 corresponds to the porous clay matrix, with a volume percentage of 60, and a permeability $K_{n m}$ given by the previous simulations. Phase 3 is the non porous minerals, with a volume percentage of 39.5 and zero permeability $K_{\text {nonporous }}=0$. The overall permeability $K_{\text {macro }}$ is obtained by solving the Darcy's equation with a variable local permeability with spatially periodic boundary conditions. The calculation yields $K_{\text {macro }} \approx 0.4 K_{n m}$, and the medium is relatively isotropic $\left(K_{\text {macroyy }} \leq K_{\text {macroxx }} \approx K_{\text {macrozz }}\right)$.

\section{Conclusion}

Compared to the mesoscopic permeability $K_{n m}$ of the combined nanoscopic and mesoscopic pore structures, the macroscopic permeability represents only $40 \%$. This means that these small scale pore structures actually drive the fluid transport through the shale. Our calculations show that the contribution of the nanoscopic pore structure cannot be neglected when assessing the transport of undisturbed matter. However, whenever the shale is cracked at the mesoscale, the permeability $K_{n m}$ is of the same order as $K_{m}$, i.e. the nanoscopic pore structure can be negelected for assessing fluid transport. It is expected that such conclusion cannot be drawn for other phenomena relating to these shale, i.e. water sorption/desorption, ion diffusion, etc.

\section{Acknowledgements}

We acknowledge funding by NEEDS MIPOR (Grand Défi Inter-Disciplinaire Nucléaire: Energie, Environnement, Déchets, Société - MIlieux POReux, funded by CNRS, CEA, EDF, ANDRA, AREVA, IRSN, BRGM, France) in 2016, under the PERMEARGILE project label.

The SEM and TEM national facilities available at the Centre Commun de Microscopie de Lille (France) are supported by the Conseil Regional du Nord-Pas de Calais, and by the European Regional Development Fund (ERDF).

\section{References}

[1] Adler P.M., Jacquin C.G., Quiblier J.A., International Journal of Multiphase Flow 16691 (1990)

[2] Desbois, G. and Urai, J.L. and Pérez-Willard, F. and Radi, Z. and van Offern, S. and Burkart, I. and Kukla, P.A. and Wollenberg, U., Journal of Microscopy 249(3), 215-235 (2013)

[3] Keller, Lukas M and Holzer, Lorenz and Wepf, Roger and Gasser, Philippe, Applied Clay Science 52, 85-95 (2011)

[4] Keller, L. M and Holzer, L. and Wepf, R. and Gasser, P. and Munch, B. and Marschall P., Physics and Chemistry of the Earth 36, 1539-1544 (2011)

[5] Keller, Lukas M. and Schuetz, Philipp and Erni, Rolf and Rossell, Marta D. and Lucas, Falk and Lucas, Miriam and Gasser, Philippe and Holzer, Lorenz, Microporous and Mesoporous Materials 170, 83-94 (2013)

[6] Marschall, P. and Horseman, S. and Gimmi, Th., Oil and Gas Science and Technology 60(1) 121-139 (2005)

[7] Mitchell, J.K., Fundamentals of Soil Behavior (2nd Edition. John Wiley and Sons, New York, 1993)

[8] Moctezuma-Berthier, A. and Vizika, O. and Thovert, J.-F. and Adler, P.M., Transport in Porous Media, 56 225-244 (2004)

[9] Pazdniakou A., Adler P.M., Advances in Water Resources, 62292 (2013)

[10] Robinet, J.C. and Sardini, P. and Coelho, D. and Parneix, J.C. and Prêt, D. and Sammartino, S. and Boller, E. and Altmann, S., Water Resources Research 48 (2012)

[11] Song Y., Davy C. A., Troadec D., Blanchenet A.-M., Skoczylas F., Talandier J., Robinet J.-C., Marine and Petroleum Geology 65, 63-82 (2015)

[12] Song Y., Davy C. A., Bertier P., Troadec D., Microporous and Mesoporous Materials 228, 64-85 (2016)

[13] Nguyen Kim T., Direct sampling applied to porous media (Université Pierre et Marie Curie, Paris, France, 2013) 1-189

[14] Yven, Béatrice and Sammartino, Stéphane and Geraud, Yves and Homand, Francoise and Villieras, Frédéric, Mémoires de la Société Géologique de France 178 73-90 (2007)

[15] Zhang, C. L. and Rothfuchs, T., Applied Clay Science 26 325-336 (2004) 